\title{
Identification and characterization of proteins, lipids, and metabolites in two organic fertilizer products derived from different nutrient sources
}

\author{
Jianyu Li', Xin Zhao ${ }^{1 *}$ D, Laura S. Bailey², Manasi N. Kamat ${ }^{2}$ and Kari B. Basso ${ }^{2}$
}

\begin{abstract}
The biochemical composition of organic fertilizers largely determines their nutrient supply characteristics following soil application as well as their potential impact on soil microbial communities. Yet, limited information is available regarding the biochemical composition of organic fertilizers derived from different nutrient sources. Here, we qualitatively analyzed the presence and abundance of proteins, lipids, and metabolites in a liquid fish fertilizer (LFF) product and a type of granular organic fertilizer (GOF) commonly used in organic vegetable production, using liquid chromatography-tandem mass spectrometry (LC-MS/MS). Our results suggest that the presence and abundance of proteins, lipids, and metabolites differ greatly between GOF and LFF. The qualitative analysis shows LFF as a rich source of metabolites, while complex proteins and long-chain saturated fatty acids are dominant in GOF. The degree of biochemical composition complexity may help explain the varying impacts of different types of organic fertilizers on nutrient availability, soil health, and environmental quality.
\end{abstract}

Keywords: Biochemical composition, LC-MS/MS, Liquid chromatography-tandem mass spectrometry, Lipidomics, Metabolomics, Nutrient availability, Organic nutrient source, Proteomics

\section{Introduction}

Organic fertilizers derived from various animal and plant-based byproducts have been widely used as nutrient sources for organic vegetable crop production. Compared to synthetic chemical fertilizers, organic fertilizers tend to have lower nutrient content along with variability in composition that depends on ingredients. However, organic fertilizers often contain beneficial microorganisms and are generally rich in organic carbon $(C)$ [1]. Applications of organic fertilizers have been shown to increase soil organic matter content, enhance overall soil enzyme activity [2], and influence microbial community

\footnotetext{
*Correspondence: zxin@ufl.edu

${ }^{1}$ Horticultural Sciences Department, University of Florida, Gainesville, FL 32611-0690, USA

Full list of author information is available at the end of the article
}

composition through addition of $\mathrm{C}$ and nitrogen (N)-rich organic compounds [3-6]. At the same time, the nutrient release process of organic fertilizers relies substantially on the complex biochemical transformation activities mediated by soil microbes [7] in addition to environmental conditions.

Early studies indicated that the general $\mathrm{C} / \mathrm{N}$ ratio and nutrient analysis of organic fertilizers might not offer sufficient information for predicting nutrient release patterns, while the biochemical composition of organic fertilizers could be highly associated with mineralization kinetics $[8,9]$. Numerous methods have been developed to estimate $\mathrm{N}$ availability in soils after organic fertilizer application, such as incubation-based $\mathrm{N}$ mineralization studies $[10,11]$. Most recently, the extractable soil protein pool was suggested to be a soil health indicator of potentially available organic N [12]. Plant metabolite 
compounds also influence soil nutrient cycling, such as $\mathrm{C}$ and $\mathrm{N}$ mineralization, through their impact on soil organisms [13]. The input of lipids, especially the longchain compounds, play an important role in soil health enhancement including the accumulation of soil organic matter content [14]. Liquid chromatography-tandem mass spectrometry (LC-MS/MS) with high-throughput capacities has been demonstrated to be a sensitive and powerful analytical tool for detecting proteins, lipids, and other metabolites from biological materials of various sources including soil [15] and plant tissue samples [1618]. However, limited information is available regarding specific biochemical compounds contained in organic fertilizers that may affect soil microbial communities and nutrient availability as well as environmental quality. LCMS/MS has rarely been used to compare biochemical compositions of organic fertilizers with different nutrient sources. Here, we chose to examine two commercially available organic fertilizer products, a liquid fish fertilizer (LFF) derived from enzymatically digested fish proteins and a granular organic fertilizer (GOF) containing feather meal and other animal waste materials, which represent commonly used nutrient sources for organic crop production [19].

Therefore, the objective of this pilot study was to employ LC-MS/MS to identify and characterize proteins, lipids, and metabolites in two commonly used commercial organic fertilizer products for qualitative comparisons of their biochemical characteristics.

\section{Materials and methods}

The LFF analyzed in this study can be used in organic fertigation systems through drip irrigation, while the GOF may be used for preplant application and/or sidedressing during the crop production season. Both organic fertilizer products have higher levels of $\mathrm{N}$ than phosphorus (P) and potassium (K) (Figure 1A; Additional file 1: Table S1). Equal amounts of LFF $(100 \mu \mathrm{L})$ and GOF $(100 \mathrm{mg})$ were individually extracted for proteins, lipids, and metabolites. The equivalent mass of $100 \mu \mathrm{L}$ LFF was determined as $97.5 \pm 1.0 \mathrm{mg}$. Lipids were extracted via a modified Folch method [20], metabolites were extracted by ice-cold methanol, and proteins by acetone/methanol precipitation. The protein pellet was reconstituted in $0.2 \%$ surfactant enhancer and concentrations were measured via a Qubit Fluorometer (Thermo Fisher Scientific Inc., Waltham, MA, USA). In-gel protein digestion was performed using trypsin as the enzyme (Promega Corporation, Madison, WI, USA). LC analysis for all was performed on a Dionex UltiMate 3000 RSLCnano system (Thermo Fisher Scientific Inc.). Lipid and metabolites were separately injected $(5 \mu \mathrm{L})$ on an Acclaim PepMap RSLC C18 column (Thermo Fisher Scientific Inc.) using acidified mobile phases of water and acetonitrile for metabolites and water, acetonitrile, and isopropanol for lipids. Proteins $(10 \mu \mathrm{g})$ were separated on a PepMAP column using acidified water and acetonitrile.

Both the lipid and metabolite compounds were analyzed on a Bruker Impact II QqTOF mass spectrometer (Bruker Daltonics, Billerica, MA, USA) using electrospray ionization operated in positive mode. Proteins were analyzed on a Thermo Scientific Q Exactive HF Orbitrap mass spectrometer equipped with an EASY Nanospray source operated in positive mode. All employed data dependent collisionally-activated dissociation. Proteomics data were analyzed in Proteome Discoverer (version 2.4) using the SEQUEST HT searching algorithm. Lipids and metabolites were analyzed in MetaboScape (version 4.0). Full experimental details are provided in Additional file 1.

\section{Results and discussion Proteomics}

Figure $1 \mathrm{~B}$ is a graphical summary of total spectral counts for each protein detected in GOF and LFF products. Hemoglobin (632 spectral counts), keratin (83 spectral counts), and actin (47 spectral counts) were the major proteins detected in GOF (862 spectral counts in total). In contrast, parvalbumin (28 spectral counts), tropomyosin (23 spectral counts), and actin (11 spectral counts) were abundant in LFF (96 spectral counts in total). Degradation of proteins in the soil can be affected by their intrinsic structures including amyloid fibril formation and glycosylation, accessibility to soil microbes or extracellular enzymes, and complex structure formation with other soil organic compounds such as tannins, lignin, and humic substances [21]. For instance, hemoglobin can bind tannins to form protein-polyphenol complex (PPC) that limits $\mathrm{N}$ mineralization due to its resistance to decomposition [22]. As a fibrous structural protein, keratin could be highly recalcitrant in response to microbial degradation because of its molecular architecture that involves formation of disulfide bonds, phosphorylation, and glycosylation [23]. The predominance of hemoglobin and keratin found in GOF might imply lower $\mathrm{N}$ mineralization rates of GOF relative to LFF.

\section{Lipidomics}

Phosphatidylcholine (PC) (e.g., C42, C44, C46) and diglyceride (DAG) (e.g., C31, C35, C37, C39, C41, C43, C47) lipids accounted for $39.4 \%$ and $26.0 \%$ of the lipids in GOF, respectively, whereas DAGs (e.g., C33, C35, C37, C39, C41, C43, C47) showed a higher classification proportion (53.9\%) in LFF (Figure 2). Our data also revealed that the majority of DAGs in both LFF and GOF contained highly polyunsaturated fatty acids (e.g., 30:4, 32:3, 

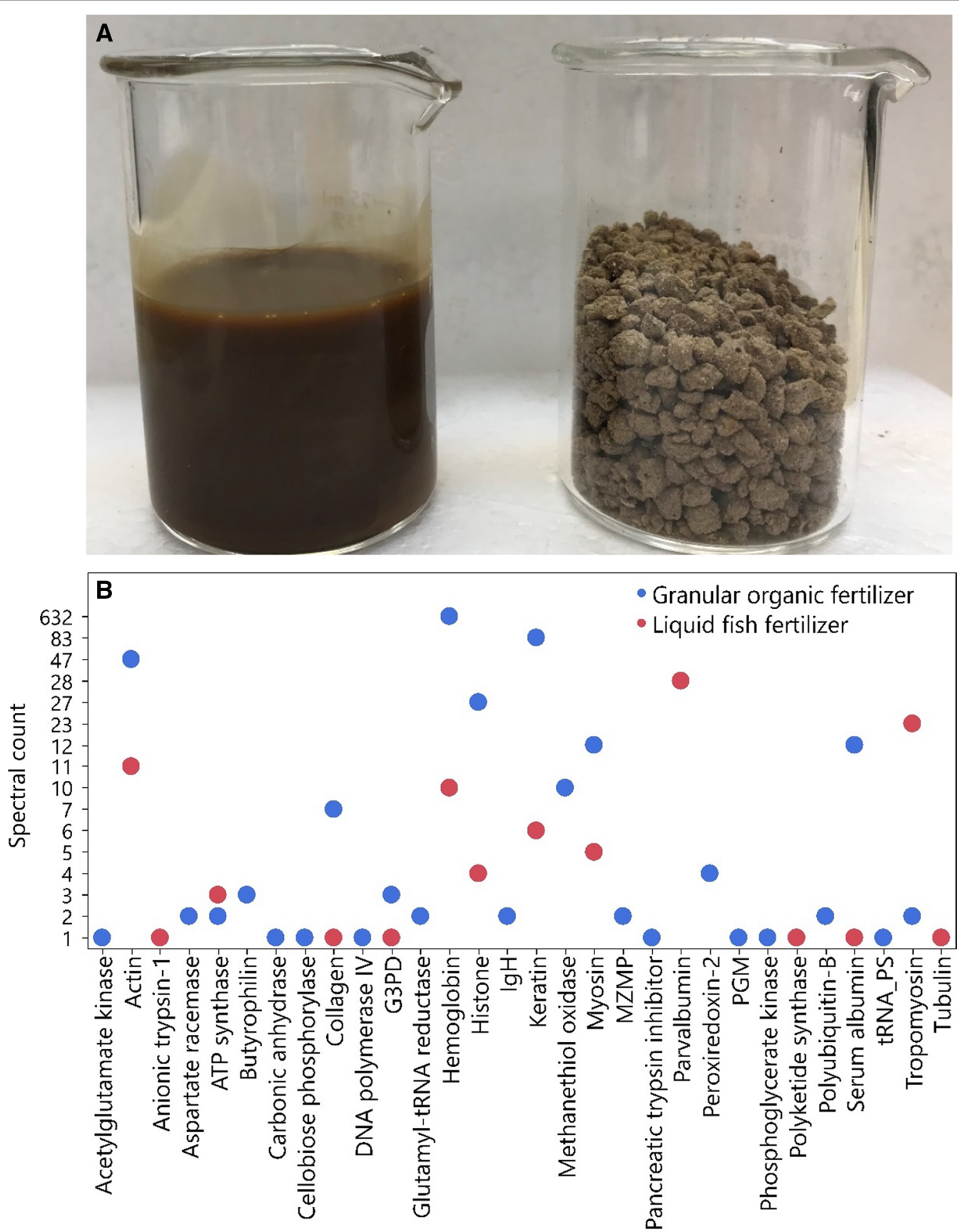

Protein

Figure 1 A Visual appearance of the two types of organic fertilizer products analyzed. Left: liquid fish fertilizer (LFF); Right: granular organic fertilizer (GOF). B Total spectral counts for each protein identified in GOF and LFF. G3PD: glyceraldehyde-3-phosphate dehydrogenase, IgH: immunoglobulin heavy chain, MZMP: mitochondrial zinc maintenance protein, PGM: phosphoglucosamine mutase, tRNA_PS: tRNA pseudouridine synthase. Y-axis scale is made disproportionally to the spectral count in order to capture the wide range of spectral counts of all proteins identified and indicate the actual values of spectral counts for each protein.

34:5, 40:9, 44:12), which could be more susceptible to microbial degradation in the soil than saturated fatty acid molecules [24]. Conversely, PC lipids containing N and P identified in GOF may degrade and release essential elements rather slowly due to its long-chain structure and the relatively high degree of fatty acid saturation. Overall, 


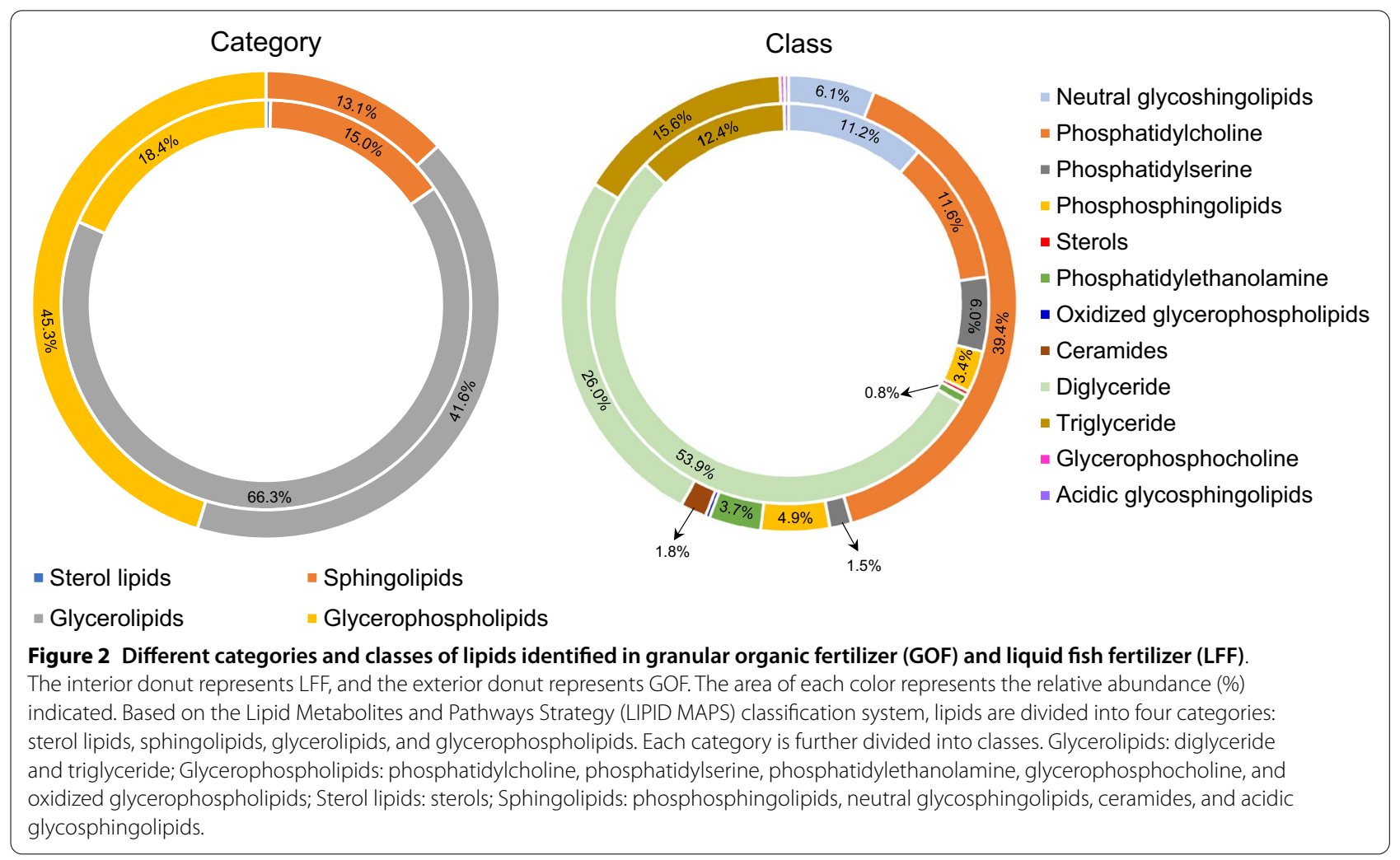

the diverse array of lipids identified in organic fertilizers in our study might indicate the potential impact of organic fertilizer application on improving soil health. For instance, lipids may serve as $C$ and energy sources for various lipid-degrading soil microorganisms, such as Bacillus, Arthrobacter, and Pseudomonas [25]. Longchain lipids $(>\mathrm{C} 20)$, in particular, are important in $\mathrm{C}$ stabilization and humification processes during the accumulation of soil organic matter [26].

\section{Metabolomics}

The identified metabolites (Additional file 1: Table S2) were categorized as amines, amides, polyols, organic acids, steroids, vitamins, isoprenoids, and plasticizers (Figure 3). Comparisons between LFF and GOF suggested that LFF is a richer source of metabolites (Figure 3 ) and the two organic fertilizers might have different impacts on soil nutrient cycling and soil microbial communities. For example, stearamide $(\mathrm{m} / z$ 284.2949; retention time (RT) $36.74 \mathrm{~min}$ ) was detected in LFF but not in GOF. It is interesting that capsaicin $(\mathrm{m} / \mathrm{z}$ 308.2218; RT $15.05 \mathrm{~min}$ ) was found in GOF but absent in LFF (Additional file 1: Table S2). Although the source of capsaicin is unclear, the GOF that contains capsaicin might potentially demonstrate a deterrent or repellent effect on certain fungi, insects, and mammals due to the irritant property of capsaicin [27]. Four fatty acid amides including pipericine $(\mathrm{m} / \mathrm{z} 336.3260$; RT $40.04 \mathrm{~min})$, macamide $(\mathrm{m} / z$ 346.3100; RT $37.08 \mathrm{~min})$, docosanamide $(\mathrm{m} / \mathrm{z}$ 340.3572; RT $42.44 \mathrm{~min})$, and erucamide $(\mathrm{m} / z$ 338.3416; RT $41.87 \mathrm{~min}$ ) appeared more abundant in LFF than GOF (Table S2), but statistical analyses with replications were not performed in this exploratory study. These amides may participate in stimulatory activities associated with soil microbial metabolism [28]. Two phenols, including $p$-coumaric acid ethyl ester $(\mathrm{m} / z$ 193.0861; RT $35.01 \mathrm{~min})$ and gingerol $(\mathrm{m} / z$ 277.1798; RT $19.20 \mathrm{~min})$, were also abundant in LFF (Table S2). As a product of acidic hydrolysis of $p$-coumaric acid ethyl ester, $p$-coumaric acid can increase soil dehydrogenase activity and abundance of soil bacterial and fungal communities [29, 30]. However, gingerol, another compound abundant in LFF, may display antimicrobial activity [31]. Additionally, given the relatively high acidity $(\mathrm{pH}=3.5)$ and abundant level of organic acids of LFF (Additional file 1: Tables S1, S2), its application could potentially result in a reduction in soil $\mathrm{pH}$ and suppression of certain soilborne pathogens in the longer term [32]. The low $\mathrm{pH}$ of LFF may be attributed to the abundance of bile acids, such as $3 \beta$-hydroxy-5cholenoic acid ( $\mathrm{m} / z$ 357.2787; RT $22.11 \mathrm{~min})$, 3-oxocholic acid ( $m / z$ 424.3060; RT $17.59 \mathrm{~min})$, cholic acid $(\mathrm{m} / z$ 426.3214; RT $18.98 \mathrm{~min})$, and nutriacholic acid $(\mathrm{m} / \mathrm{z}$ 


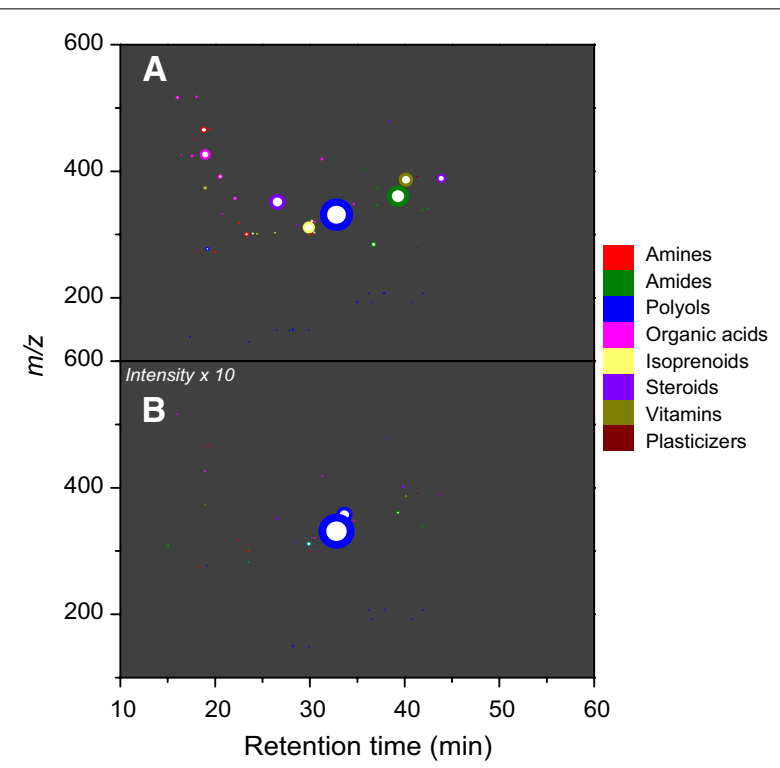

Figure 3 Survey overview of metabolite presence and relative abundance broken down by class and functionality for liquid fish fertilizer (A) and granular organic fertilizer (B). These compounds were annotated and categorized with the help of PubChem based on the general chemical structures of compounds. The compound abundance was determined using the spectral and analyte metabolite matches compiled in MetaboScape. The circle color represents compound category and circle size is proportional to the compound abundance. The abundance values were scaled up by an intensity factor of 10 in plot $B$ in order to make dots visible in plot B.

391.2844; RT $20.55 \mathrm{~min}$ ) (Table S2). Moreover, bacterial degradation products (e.g., androstadienediones) of bile acids could also pose a potential risk, such as reduced reproduction rates, to invertebrates in agricultural soils [33]. Similarly, as a steroid hormone, progesterone $(\mathrm{m} / \mathrm{z}$ 315.2319; RT $28.67 \mathrm{~min}$ ), which was discovered in both LFF and GOF (Additional file 1: Table S2), might accumulate in soil and cause adverse impacts on the environment [34].

\section{Conclusions}

Our qualitative analysis is the first study attempting to elucidate the biochemical composition of organic fertilizers, explore its linkage to nutrient availability of organic fertilizers, and envision its impact on soil quality and health. The dominance of complex proteins and long-chain saturated fatty acids contained in GOF suggests that GOF might decompose and release nutrients at a slower rate in the soil relative to LFF. A diverse variety and abundance of metabolites were identified in GOF and LFF, indicating potentially different impacts of these organic fertilizers on soil microbial communities and nutrient availability. Future research can examine more organic fertilizer products derived from various nutrient sources and include quantitative analysis for in-depth comparisons. Overall, our study demonstrates the complexity in biochemical composition of organic fertilizers and suggests the need to further understand how organic fertilizers with different biochemical profiles influence nutrient cycling, soil health, and environmental quality.

\section{Abbreviations \\ LFF: Liquid fish fertilizer; GOF: Granular organic fertilizer; GC/MS: Gas chroma- tography-mass spectrometry; LC-MS/MS: Liquid chromatography-tandem mass spectrometry; PPC: Protein-polyphenol complex; PC: Phosphatidylcho- line; DAG: Diglyceride.}

\section{Supplementary Information}

The online version contains supplementary material available at https://doi. org/10.1186/s13765-021-00625-2.

Additional file 1: Table S1. Basic characteristics of the two organic fertilizer products used in this study. Table S2. List of metabolites identified by LC-MS/MS in liquid fish fertilizer and granular organic fertilizer.

\section{Acknowledgements}

This work was supported by Organic Agriculture Research and Extension Initiative grant no. 2015-51300-24134 from the USDA National Institute of Food and Agriculture. We thank James Colee for his assistance with data analyses. We also thank the Mass Spectrometry Research and Education Center at the University of Florida and their funding source: NIH S10 OD021758-01A1.

\section{Authors' contributions}

$J L$ and $X Z$ designed the experiment and wrote the manuscript. LSB, MNK, and KBB performed the LC-MS/MS analysis and helped with data analysis and interpretation. All authors read and approved the final manuscript.

\section{Funding}

Funding was received from the Organic Agriculture Research and Extension Initiative (Grant No. 2015-51300-24134) funded by the USDA National Institute of Food and Agriculture.

Availability of data and materials

All data generated or analyzed during the present study are included in this published article.

\section{Declarations}

Competing interests

There is no conflict of interest.

\section{Author details}

${ }^{1}$ Horticultural Sciences Department, University of Florida, Gainesville, FL 32611-0690, USA. ² Department of Chemistry, University of Florida, Gainesville, FL 32611-7200, USA.

Received: 13 April 2021 Accepted: 13 July 2021

Published online: 05 October 2021

References

1. Timsina J (2018) Can organic sources of nutrients increase crop yields to meet global food demand? Agronomy 8:214

2. Bowles TM, Acosta-Martínez V, Calderón F, Jackson LE (2014) Soil enzyme activities, microbial communities, and carbon and nitrogen availability 
in organic agroecosystems across an intensively-managed agricultural landscape. Soil Biol Biochem 68:252-262

3. Burger M, Jackson LE (2003) Microbial immobilization of ammonium and nitrate in relation to ammonification and nitrification rates in organic and conventional cropping systems. Soil Biol Biochem 35:29-36

4. Lin Y, Ye G, Kuzyakov Y, Liu D, Fan J, Ding W (2019) Long-term manure application increases soil organic matter and aggregation, and alters microbial community structure and keystone taxa. Soil Biol Biochem 134:187-196

5. Martínez-Alcántara B, Martínez-Cuenca MR, Bermejo A, Legaz F, Quiñones A (2016) Liquid organic fertilizers for sustainable agriculture: nutrient uptake of organic versus mineral fertilizers in citrus trees. PLoS ONE 11:e0161619

6. Tian J, Lou Y, Gao Y, Fang H, Liu S, Xu M, Blagodatskaya E, Kuzyakov Y (2017) Response of soil organic matter fractions and composition of microbial community to long-term organic and mineral fertilization. Biol Fertil Soils 53:523-532

7. Jacoby R, Peukert M, Succurro A, Koprivova A, Kopriva S (2017) The role of soil microorganisms in plant mineral nutrition—current knowledge and future directions. Front Plant Sci 8:1617

8. McGill WB, Hunt HW, Woodmansee RG, Reuss JO, Paustian KH (1981) Formulation, process controls, parameters and performance of PHOENIX: a model of carbon and nitrogen dynamics in grassland soils. In: Frissel MJ van Veen JA (eds) Simulation of nitrogen behaviour of soil-plant systems. Centre for Agricultural Publishing and Documentation, Wageningen, The Netherlands, pp 171-191

9. Paré T, Dinel H, Schnitzer M, Dumontet S (1998) Transformations of carbon and nitrogen during composting of animal manure and shredded paper. Biol Fertil Soils 26:173-178

10. Cassity-Duffey K, Cabrera M, Gaskin J, Franklin D, Kissel D, Saha U (2020) Nitrogen mineralization from organic materials and fertilizers: Predicting $N$ release. Soil Sci Soc Am J 84:522-533

11. Hartz TK, Johnstone PR (2006) Nitrogen availability from high-nitrogencontaining organic fertilizers. HortTechnology 16:39-42

12. Hurisso TT, Moebius-Clune DJ, Culman SW, Moebius-Clune BN, Thies JE, van Es HM (2018) Soil protein as a rapid soil health indicator of potentially available organic nitrogen. Agric Environ Lett 3:180006

13. Norris CE, Preston CM, Hogg KE, Titus BD (2011) The influence of condensed tannin structure on rate of microbial mineralization and reactivity to chemical assays. J Chem Ecol 37:311-319

14. Bull ID, van Bergen PF, Nott CJ, Poulton PR, Evershed RP (2000) Organic geochemical studies of soils from the Rothamsted classical experiments$\checkmark$. The fate of lipids in different long-term experiments. Org Geochem 31:389-408

15. Nguyen TD, Lesani M, Forrest I, Lan Y, Dean DA, Gibaut QMR, Guo Y, Hossain E, Olvera M, Panlilio H, Parab AR, Wu C, Bernatchez JA, Cichewicz RH, McCall LI (2020) Local phenomena shape backyard soil metabolite composition. Metabolites 10:86

16. Parejo I, Jauregui O, Sánchez-Rabaneda F, Viladomat F, Bastida J, Codina C (2004) Separation and characterization of phenolic compounds in fennel (Foeniculum vulgare) using liquid chromatography-negative electrospray ionization tandem mass spectrometry. J Agric Food Chem 52:3679-3687

17. Nouri MZ, Komatsu S (2010) Comparative analysis of soybean plasma membrane proteins under osmotic stress using gel-based and LC MS/ MS-based proteomics approaches. Proteomics 10:1930-1945

18. Della Corte A, Chitarrini G, Di Gangi IM, Masuero D, Soini E, Mattivi F, Vrhovsek U (2015) A rapid LC-MS/MS method for quantitative profiling of fatty acids, sterols, glycerolipids, glycerophospholipids and sphingolipids in grapes. Talanta 140:52-61

19. Gaskell M, Smith R (2007) Nitrogen sources for organic vegetable crops. HortTechnology 17:431-441

20. Folch J, Lees M, Stanley GHS (1957) A simple method for the isolation and purification of total lipides from animal tissues. J Biol Chem 226:497-509

21. Rillig MC, Caldwell BA, Wösten HAB, Sollins P (2007) Role of proteins in soil carbon and nitrogen storage: controls on persistence. Biogeochemistry 85:25-44

22. Hong JK, Jho EH, Choi HS, Kang G (2018) Role of hemoglobin in hemoglobin-based remediation of the crude oil-contaminated soil. Sci Total Environ 627:1174-1181

23. Lange L, Huang Y, Busk PK (2016) Microbial decomposition of keratin in nature - a new hypothesis of industrial relevance. Appl Microbiol Biotechnol 100:2083-2096

24. He Y, Sun C, Li W, Yang GP, Ding H (2018) Degradation of lipids in seasonal hypoxic seawater under different oxygen saturation. J Ocean Limnol 36:1570-1585

25. Souza RC, Cantão ME, Nogueira MA, Vasconcelos ATR, Hungria M (2018) Outstanding impact of soil tillage on the abundance of soil hydrolases revealed by a metagenomic approach. Braz J Microbiol 49:723-730

26. de Assis CP, González-Vila FJ, Jucksch I, González-Pérez JA, Neves JCL, Lani JL, de Sá Mendonça E (2011) Lipid abundance and composition of a humic Oxisol as a function of land use. Sci Agric 68:230-236

27. Tewksbury JJ, Reagan KM, Machnicki NJ, Carlo TA, Haak DC, Peñaloza ALC, Levey DJ (2008) Evolutionary ecology of pungency in wild chilies. Proc Natl Acad Sci USA 105:11808-11811

28. Sun L, Lu Y, Kronzucker HJ, Shi W (2016) Quantification and enzyme targets of fatty acid amides from duckweed root exudates involved in the stimulation of denitrification. J Plant Physiol 198:81-88

29. Wang ML, Gu Y, Kong CH (2008) Effects of rice phenolic acids on microorganisms and enzyme activities of non-flooded and flooded paddy soils. Allelopathy J 22:311-319

30. Zhou X, Wu F (2012) p-Coumaric acid influenced cucumber rhizosphere soil microbial communities and the growth of Fusarium oxysporum f.sp. cucumerinum Owen. PLOS ONE 7:e48288

31. Beristain-Bauza SDC, Hernández-Carranza P, Cid-PérezTS, Ávila-Sosa R, Ruiz-López II, Ochoa-Velasco CE (2019) Antimicrobial activity of ginger (Zingiber Officinale) and its application in food products. Food Rev Int 35:407-426

32. Abbasi PA, Lazarovits G, Jabaji-Hare S (2009) Detection of high concentrations of organic acids in fish emulsion and their role in pathogen or disease suppression. Phytopathology 99:274-281

33. Mendelski MN, Dölling R, Feller FM, Hoffmann D, Fangmeier LR, Ludwig KC, Yücel O, Mährlein A, Paul RJ, Philipp B (2019) Steroids originating from bacterial bile acid degradation affect Caenorhabditis elegans and indicate potential risks for the fauna of manured soils. Sci Rep 9:11120

34. Zhang FS, Xie YF, Li XW, Wang DY, Yang LS, Nie ZQ (2015) Accumulation of steroid hormones in soil and its adjacent aquatic environment from a typical intensive vegetable cultivation of North China. Sci Total Environ 538:423-430

\section{Publisher's Note}

Springer Nature remains neutral with regard to jurisdictional claims in published maps and institutional affiliations. 